\title{
Position-Induced Phase Change in a TASEP with a Double-Chain Section (a Model of Biological Transport)
}

\author{
Nina C. Pesheva* and Jordan G. Brankov ${ }^{\dagger}$ \\ *Institute of Mechanics-BAS, Sofia, Bulgaria \\ Email: nina@imbm.bas.bg \\ $\dagger$ Joint Institute for Nuclear Research, Dubna, Russian Federation \\ Email: brankov@theor.jinr.ru
}

Received: 31 August 2012, accepted: 21 November 2012, published: 21 December 2012

\begin{abstract}
The totally asymmetric simple exclusion processes (TASEP) has been used since 1968 to model different biochemical processes, including kinetics of protein synthesis, molecular motors traffic, collective effects in genetic transcription. Here, we consider TASEP defined on an open network consisting of simple head and tail chains with a double-chain section in-between. Our results of Monte Carlo simulations show a novel property of the model when the simple chains are in the maximum-current phase: upon moving the double-chain defect from the central position forward or backward along the network, keeping fixed the length of both the defect and the whole network, a position-induced phase change in the parallel defect chains takes place. This phenomenon is explained in terms of finite-size dependence of the effective injection and removal rates at the ends of the double-chain defect. Some implications of the results for molecular motors cellular transport along such networks are suggested. However, at present these are just speculations which need further examinations.
\end{abstract}

Keywords-TASEP; Molecular motors traffic; kinetics of protein synthesis; traffic flow models; non-equilibrium phase transitions; non-equilibrium statistical physics

\section{INTRODUCTION}

The world of non-equilibrium phenomena is more diverse and much more interesting as compared to our experience with its equilibrium counterpart. Rigor- ously put, true equilibrium phenomena are an idealization which is seldom met in nature. The development of a fundamental and comprehensive understanding of physics far from equilibrium is currently under way. That is why the study of simple non-equilibrium models like the totally asymmetric simple exclusion process (TASEP) (see, e.g., [1, 2, 3, 4, 5, 6, 7, 8, 9]) is very informative and helpful. This approach-the study of simple model systems, has shown to be very effective in the equilibrium statistical mechanics and now it is intensively exploited also in the non-equilibrium case. One can see that recently more methods and concepts from non-equilibrium statistical physics are applied to model processes in living systems and biological phenomena $[10,11,12,13,14,15,16,17,18]$. This is quite natural since the object of non-equilibrium statistical physics are open many-particle systems with macroscopic currents of energy and/or particles. Biological systems, on the other hand, are rather complex systems which in order to function properly need energy and matter flows. There are biological transport phenomena which can be considered to be restricted to an effectively one-dimensional track, e.g., stepping of kinesins and dyneins along microtubules, translocation of RNA polymerase (RNAP) on DNA during transcription, ribosomes on messenger RNA (mRNA) during protein syntheses - a process referred to as translation. Kinesins and dyneins are cytoskeletal 
motors: kinesin moves cargo inside cells away from the nucleus along microtubules and dynein transports cargo along microtubules towards the cell nucleus. All these stochastic processes are of special interest due to their fundamental importance for the functioning of living cells. Hence, they are a challenging object for mathematical modeling and discrete stochastic models seem adequate for that purpose. Usually a large number of such agents move unidirectionally along the same track with excluded volume interaction, which makes the simple models of vehicular traffic appropriate for incorporation in more sophisticated ones. For example, stochasticity and traffic jams in the transcription of ribosomal RNA have been considered by Klumpp and Hwa [14]. In the present study, we are concerned with one specific example of application of a simple non-equilibrium model, the TASEP, to the protein synthesis. Since 1968 this model has been used to model different biological processes [10, 11, 12, 13, 14, 15, 16, 17, 18] including the phenomenon of protein synthesis [10]. In the last twenty years, the non-equilibrium statistical physicists [19, 20, 21, 22, 23, 24] are very much interested in the study of different kind of models which are expected to provide deep understanding of the generic behavior of non-equilibrium systems. Another challenging problem, from both biological and mathematical point of view, is the consideration of biochemical transport phenomena on networks with non-trivial topology. Our goal here is to present a study of the effects, arising in TASEP, defined on a simple example of such a network: a linear chain of attachment sites with a double-chain defect inserted in it [25]. For other studies of TASEP on topologies more complex than a single segment see [26, 27, 28, 29]. Recently, applications to biological transport have motivated generalizations of the TASEP to cases when the entry rate is chosen to depend on the number of particles in the reservoir (TASEP with finite resources) [30, 31]. This year, the cases of multiple competing TASEPs with a shared reservoir of particles [32, 33], and TASEP with Langmuir kinetics and memory reservoirs [34] were studied too. The next section is devoted to the single chain TASEP, then a short overview is given on the TASEP with a double-chain section in-between [25]. The last section is devoted to our new Monte Carlo simulation results displaying a novel property of the model with the double-chain section in the maximum-current phase.

\section{Model AND APPLiCATIONS}

\section{A. Single Chain TASEP}

One of the simplest driven (non-equilibrium) models of many-particle systems with particle conserving stochastic dynamics is the asymmetric simple exclusion process (ASEP). It has been extensively studied on simple chains with periodic, closed and open boundary conditions. In the extremely asymmetric case particles are allowed to move with in one direction only - this is the totally asymmetric simple exclusion process (TASEP). It was first introduced in [10] as a model of protein synthesis; in the context of interacting Markov processes, see [1]. Its steady states are exactly known for both open and periodic boundary conditions, for continuous-time and several kinds of discrete-time dynamics. Here we shall focus our attention on the steady states of the open TASEP with continuous-time stochastic dynamics on a simple chain, illustrated in Fig. 1. For a review on the exact results for the stationary states of TASEP under different kinds of stochastic dynamics, and its numerous applications, we refer the reader to [4, 22].

The continuous-time dynamics is modeled by the so called random-sequential update: in the algorithm one chooses with equal probability any one of the lattice sites (the left reservoir is included as an additional site), and, if the chosen site is occupied by a particle, moves it (with rate $p=1$ ) to the nearest-neighbor site on the right, provided the target site is empty. In the case of open system, particles are injected at the left end with rate $\alpha$ and removed at the right end with rate $\beta$ when the last site is occupied. When $\alpha, \beta \in(0,1]$ the boundary conditions correspond to coupling of the system to reservoirs of particles with constant densities $\alpha$ and $1-\beta$, respectively.

As predicted by Krug [21], the change of the boundary rates induces non-equilibrium phase transitions between different stationary phases. In the thermodynamic limit, the phase diagram of the stationary states in the plane of the particle injection and removal rates is shown in Fig. 2. It exhibits three distinct phases: a low-density freeflow phase (region $A I \cup A I I$ ), a high-density congested traffic one (region $B I \cup B I I$ ), and a maximum current phase (region $M C$ ), characterized by a synchronized flow in which jams and free-flow coexist at intermediate densities. These phases are separated by lines of non-equilibrium first-order and second-order phase transitions. Here we need to mention some basic facts obtained in the case of continuous-time dynamics: (a) the correlations in the bulk of an infinite chain vanish 


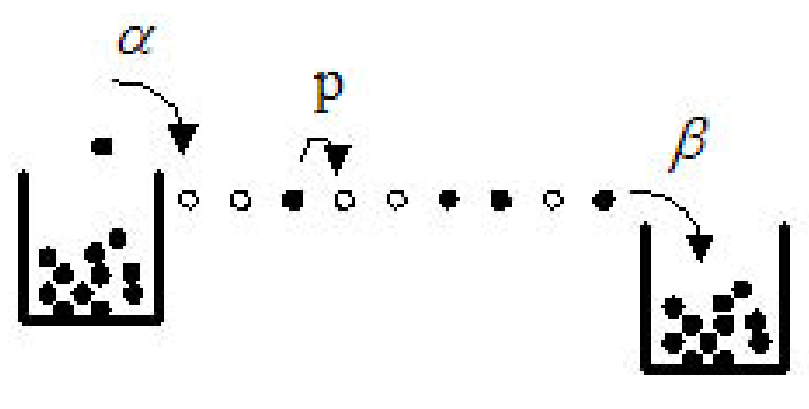

Fig. 1. Schematic representation of the open TASEP on a simple chain; for details see text.

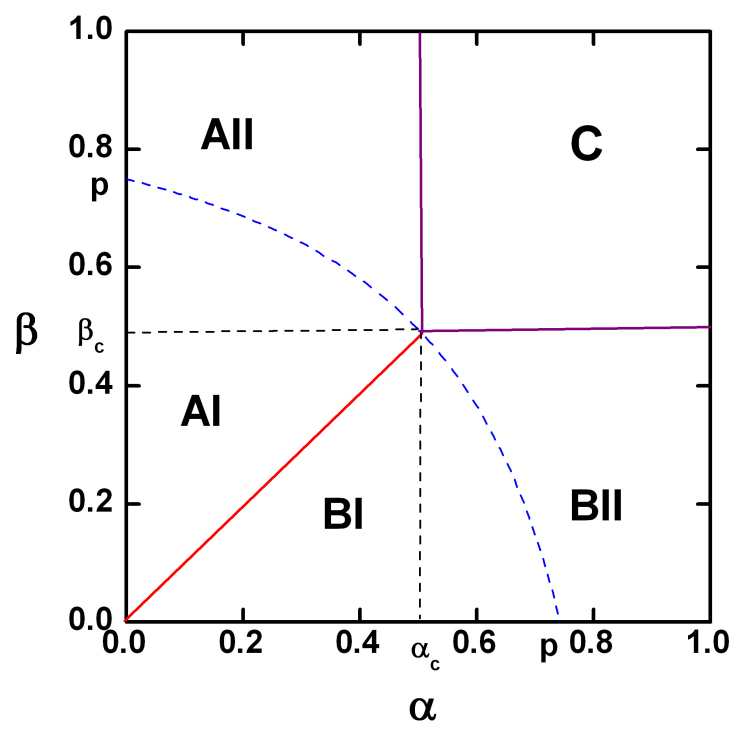

Fig. 2. Phase diagram of the open TASEP on a simple chain. The regions of the different phases are explained in the text.

and the dependence of the stationary current of particles $J$ on the average density $\rho$ is given by $J=\rho(1-\rho)$; (b) in the maximum current phase $\rho=1 / 2$ and $J=1 / 4$; when $J<1 / 4$, there are two densities which support that current:

$$
\rho_{ \pm}(J)=\left[1 \pm(1-4 J)^{1 / 2}\right] / 2,
$$

$\left(\rho_{-}\right)$is the bulk density in the low-density (high-density) phase.

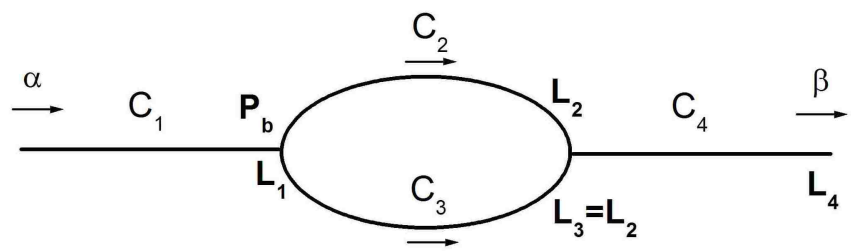

Fig. 3. Schematic representation of the network: a single chain with a two-chain incertion. The segments $C_{2}$ and $C_{3}$ have equal length $L_{2}\left(L_{3}=L_{2}\right)$ in the case under consideration. The particles are injected at the left end with a rate $\alpha$ and removed at the right end with a rate $\beta$. The particles move from left to right, at the branching point $P_{b}=L_{1}$ they take with equal probability the upper or the lower branch.

\section{B. TASEP with a Double-Chain Section}

The idea of studying networks, composed of chain segments, which exhibit the bulk behavior of an open TASEP under boundary conditions given in terms of effective input and output rates, was first advanced in our work [25]. The network considered there is shown schematically in Fig. 3. The appearance of correlation effects, close to the ends of the chain segments, as well as of cross-correlations in the double-chain segment was found.

The same approach was applied in Ref. [26] to an open network consisting of one vertex with two incoming chains, coupled to one reservoir, and one outgoing chain, coupled to another reservoir. Different versions of simple networks were studied also in Refs. [25] and [26]. In the latter work the notion of particle-hole symmetry in the presence of a junction was carefully analyzed and an appropriate interpretation on the microscopic level was given. TASEP with parallel update on single multipleinput-single-output junctions has been investigated too [29]. The main concern in the above works was the construction of the phase diagram under different open boundary conditions.

Here we continue the investigation of the network considered in [25], see Fig. 3. Note, that the last site $i=L_{1}$ of the head chain is a branching point, from which the particles can take the upper or the lower branch of the two-chain section with equal probability. Simultaneous and independent traffic of particles on the two equivalent branches was simulated. The parallel branches merge at site $i=L_{1}+L_{2}$, where the particles have to wait for the first site of the tail chain $i=L_{1}+L_{2}+1$ to become empty. We have denoted the phase structure of the model by $\left(X_{1}, X_{2,3}, X_{4}\right)$, where $X_{k}(k=1,2,3,4)$ 
stands for one of the stationary phases of the chain segment $C_{k}: L D$ - low density, $H D$-high density, $M C$ maximum current, and $C L-$ coexistence line. Our analytical analysis of the allowed phase structures, based on the properties of single chains in the thermodynamic limit, and the neglect of the pair correlations between the nearest-neighbor occupation numbers at the junctions of different chain segments, yielded 8 possibilities. Here we focus our investigation on 3 of the most interesting cases $(M C, L D, M C),(M C, C L, M C)$, and $(M C, H D, M C)$, which appear under the conditions $\alpha>1 / 2, \beta>1 / 2$, corresponding to the maximum current phase of a single chain. The phase state of the chains in the double-chain defect depends on the effective injection rate $\alpha^{*}$ of particles at the first site of each of the chain segments $C_{2,3}$ and on the effective removal rate $\beta^{*}$ of particles from the last site of each of these chains. As in the case of a single infinite chain, the density profiles of $C_{2}$ and $C_{3}$ are similar to the ones in the $L D, C L$, and $H D$ phases when $\beta^{*}<\alpha^{*}<1 / 2$, $\beta^{*}=\alpha^{*}<1 / 2$, and $\alpha^{*}<\beta^{*}<1 / 2$, respectively. The crucial difference now is that the above effective rates depend on the finite size of the head and tail simple chain segments.

In the present interpretation, the hard-core particles represent individual molecular motors.

\section{Results AND Discussion}

As a result of Monte Carlo simulations we have found a novel property of the model in the maximum-current phase, i.e., when $\alpha>1 / 2$ and $\beta>1 / 2$. Then the current $J_{2,3}$ trough each of the chains $C_{2,3}$ equals half of the maximum current, i.e., $J_{2,3}=1 / 8$. Therefore, due to the fundamental relationship $J=\rho(1-\rho)$, in the thermodynamic limit these chains can be found either in a low-density phase with bulk density

$$
J(1 / 8)=[1-\sqrt{0.5}] / 2 \approx 0.14645,
$$

or in the high-density phase with bulk density

$$
J(1 / 8)=[1+\sqrt{0.5}] / 2 \approx 0.85355,
$$

or on the coexistence line of these two phases. Upon moving the double-chain defect along the network, keeping fixed the lengths of both the defect and the whole network, a position-induced phase change in the defect chains takes place. This change from the coexistence line to a low- or high-density phase is observed in the density profile of each of the chains forming the defect.

In Fig. 4 we show our simulation results for the density distributions for a rather small system of fixed

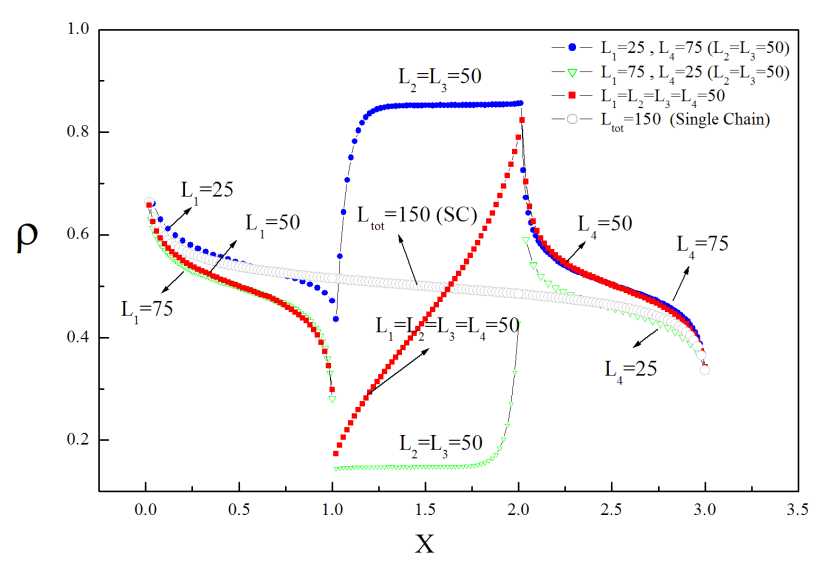

Fig. 4. Simulation results: density profiles as a function of the scaled distance $x=i / L_{k}$, for the system with the $(M C, C L, M C)$ phase structure, appearing when . The symmetric case with $L_{1}=$ $L_{2,3}=L_{4}=50$ is shown with red squares. The change of the density profiles in the double-chain section is clearly seen: when $L_{1}=25, L_{4}=75$ its shape is characteristic of the $H D$ phase (blue circles); when $L_{1}=75$ and $L_{4}=25$ its shape is characteristic of the $L D$ phase (green triangles).

total length $L_{t o t}=L_{1}+L_{2,3}+L_{4}=150$ sites and fixed size of the double-chain section, $L_{2}=L_{3}=50$. The ensemble averaging was performed over 200 independent runs and after 3000000 Monte Carlo steps were omitted in order to ensure that the system had reached a stationary state. One can easily see the sharp change, which the density profiles undergo, when the position of the loop is shifted. As a reference, the results for the density profiles of the system with segments of equal length $L_{1}=L_{2,3}=L_{4}=50$ are shown with red squares. Grey circles In the latter case the two branches of the defect section are on the coexistence line. However, when the head chain is shorter, e.g., when $L_{1}=25$ and, respectively, $L_{4}=75$, the density distribution in the double-chain section is typical for the $H D$ phase (see the results shown with blue circles). In the opposite case, when the head chain is longer than the tail one, $L_{1}=75$ and $L_{4}=25$, the density distribution of the double chain-section has the typical shape of the $L D$ phase (shown with green triangles).

The spatial behavior of the correlations between nearest-neighbor occupation numbers, shown in Figure 5 , is also typical for the corresponding phases.

An explanation of the phenomenon can be given in terms of finite-size dependence of the effective injection and removal rates at the ends of the double-chain defect. In the symmetric case, when $L_{1}=L_{4}$, we observe 


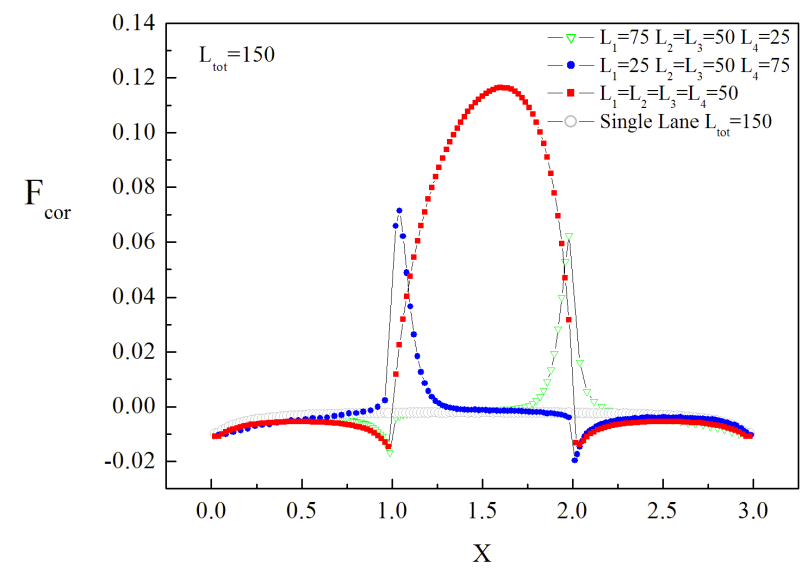

Fig. 5. Simulation results: Nearest-neighbor correlations $F_{\text {corr }}$, in the $(M C, C L, M C)$ phase state of the system, as a function of the scaled distance $x=i / L_{k}$, for different positions of the double-chain segment.

$\alpha^{*} \approx \beta^{*}$ and, in the thermodynamic limit, the defect chains should be on the coexistence line. This fact is demonstrated by the (almost) linear density profile, changing from $\rho_{-}(1 / 8)$ at the left end to $\rho_{+}(1 / 8)$ at the right end. Such a linear profile is known to result from a freely moving domain wall separating the low-density and high-density regions. Due to the size dependence of the effective rates, on moving the defect to the left (i.e., when $L_{1}$ decreases and $L_{4}$ increases, so that $L_{1}+L_{4}$ remains constant), $\alpha^{*}$ increases and $\beta^{*}$ slightly decreases, thus the condition $\alpha^{*}>\beta^{*}$ becomes fulfilled and the chains $C_{2,3}$ obtain a density profile, characteristic of the high-density phase. In the opposite case, on moving the defect to the right (i.e., when $L_{1}$ increases and $L_{4}$ decreases, so that $L_{1}+L_{4}$ is constant), $\alpha^{*}$ slightly decreases and $\beta^{*}$ increases, so that the condition $\alpha^{*}<\beta^{*}$ takes place and the chains $C_{2,3}$ obtain a density profile, characteristic of the low-density phase.

It is interesting to note, that the average velocity of particles $v$, defined from the relation $J=\rho v$, is higher (lower) in the low-density (high-density) phase than in the head and tail chains, for which $v_{M C}=1 / 2$. Indeed, in the $L D$ phase

$$
v_{L D}=1 /[4(1-\sqrt{0.5})] \approx 0.85355,
$$

and in the $H D$ phase

$$
v_{H D}=1 /[4(1+\sqrt{0.5})] \approx 0.14645,
$$

Another notable observation is, that not only the bulk density of a single chain in the double-chain segment in the $L D(H D)$ phase is lower (higher) than the bulk density of the head and tail chains, for which $\rho_{M C}=$ $1 / 2$, but the same relation holds for the sum of the bulk densities of both chains in the double-chain segment. Indeed, in the $L D$ phase

$$
2 \rho_{-}(1 / 8)=1-\sqrt{0.5} \approx 0.29289,
$$

and in the $H D$ phase

$$
2 \rho_{+}(1 / 8)=1+\sqrt{0.5} \approx 1.7071,
$$

In general, for a multi-chain defect, consisting of $\mathrm{n}$ parallel identical chains, in the $L D$ phase we obtain for the total bulk density of particles in the defect

$n \rho_{ \pm}(1 / 4 n)=n\left[1 \pm\left(1-n^{-1}\right)^{1 / 2}\right] / 2 \rightarrow 1 / 4, n \rightarrow \infty$

Therefore, the unlimited increase of the number of chains in the defect part, tends to lower the total bulk density of particles in it from $2 \rho_{-}(1 / 8) \approx 0.29289$ down to 0.25 . This is a very interesting and useful property.

\section{CONCLUSION}

A possible biochemical interpretation of the model, considered here, can be given in terms of molecular motors moving along linear biopolymers, such as actin filaments, microtubules, DNA and RNA molecules. Our model ignores the possibility of backward steps, as well as the initiation stage, the dissociation from the track and the sequence of intermediate biochemical states, for example, the arrival and binding of a fuel molecule. We have focused on the effect of a non-trivial topology on the transport of hard-core particles. As pointed out by Pronina and Kolomeisky [26], the realistic description of cellular transport, requires also to include the possibility of motion on lattices with a more complex geometry. For example, there are indications, that the number of proto-filaments, that kinesins walk on, may vary in the microtubules. This indicates the existence of junctions and other lattice defects, which may be responsible for some human diseases. The network with a double-chain defect, considered by us, can be thought of as some sort of genetic malformation or defect, caused by radiation or some other source. Our main results concern the bulk density and the average velocity of particles in the defect chains, in the regime of maximum current through the whole network. One can imagine scenarios, when it is needed to minimize or maximize some of the above mechanical parameters, presumably, for engineering novel cellular behavior. Then some hints from models of traffic on tracks with parallel sections could 
be helpful. From the point of view of statistical physics, one is interested in a number of issues. A fundamental question concerns the "stability" of steady-state properties with respect to model modifications. Which changes of the microscopic model details will lead to changes of the macroscopic behavior? Also, while for equilibrium systems basic notions of universality and independence from dynamic details are well understood, only initial steps are taken towards extending these notions towards non-equilibrium systems and more specifically towards non-equilibrium steady states [35, 36]. We would like to conclude by pointing out that even though such simple models may not permit immediate comparisons with available experimental data, due to the significant amount of simplification and/or abstraction involved, they can still be quite useful in guiding future experimental work.

\section{ACKNOWLEDGMENT}

N.P. acknowledges a financial support by the project BG051PO001/3.3-05.001 "Science and Business", financed by the Operational Programme "Human Resources Development" of ESF, under contract number D02-780/28.12.2012.

\section{REFERENCES}

[1] F. Spitzer, "Interaction of Markov processes", Adv. Math. 5, 246 (1970). http://dx.doi.org/10.1016/0001-8708(70)90034-4

[2] B. Derrida, M. R. Evans, V. Hakim, and V. Pasquier, "Exact solution of a 1D asymmetric exclusion model using a matrix formulation", J. Phys. A 26, 1493-1517 (1993). http://dx.doi.org/10.1088/0305-4470/26/7/011

[3] H. Hinrichsen, "Matrix product ground states for exclusion processes with parallel dynamics", J. Phys. A 29, 3659-3667 (1996). http://dx.doi.org/10.1088/0305-4470/29/13/030

[4] N. Rajewsky, L. Santen, A. Schadschneider, and M. Schreckenberg, "The Asymmetric Exclusion Process: Comparison of Update Procedures", J. Stat. Phys. 92, 151-194 (1998). http://dx.doi.org/10.1023/A:1023047703307

[5] M. R. Evans, N. Rajevsky, and E. R. Speer, "Exact Solution of a Cellular Automaton for Traffic", J. Stat. Phys. 95, 45-96 (1999). http://dx.doi.org/10.1023/A:1004521326456

[6] J. Brankov, N. Pesheva, N. Valkov, "Exact results for a fully asymmetric exclusion process with sequential dynamics and open boundaries", Phys.
Rev. E 61, 2300-2318 (2000). http://dx.doi.org/10.1103/PhysRevE.61.2300

[7] J. Brankov, N. Pesheva, N. Bunzarova, "OneDimensional Traffic Flow Models: Theory and Computer Simulations", a cond-mat prepint: arXiv:0803.2625 (2008) (at http://arxiv.org ).

[8] M. Schreckenberg, A. Schadschneider, K. Nagel, N. Ito, "Discrete stochastic models for traffic flow", Phys. Rev. E 51, 2939-2949 (1995).

[9] D. Helbing, "Traffic and related self-driven manyparticle systems", Rev. Mod. Phys. 73, 1067-1141 (2001). http://dx.doi.org/10.1103/RevModPhys.73.1067

[10] C. T. MacDonald, J. H. Gibbs, and A. C. Pipkin, Kinetics of biopolymerization on nucleic acid templates. Biopolymers 6, 1-25 (1968).

[11] L. B. Shaw, R. K. P. Zia, K. H. Lee, "Totally asymmetric exclusion process with extended objects: A model for protein synthesis", Phys. Rev. E 68, 021910 (1-17) (2003).

[12] D. Chowdhury, A. Schadschneider, K. Nishinary, "Physics of Transport and Traffic Phenomena in Biology: from Molecular Motors and Cells to Organisms", Phys. Life Reviews 2, 318-352 (2005); arXiv: physics/050925.

[13] A. B. Kolomeisky, M. E. Fisher, "Molecular motors: a theorist's perspective", Annu. Rev. Phys. Chem. 58, 675-695 (2007).

[14] S. Klumpp, T. Hwa, "Stochasticity and traffic jams in the transcription of ribosomal RNA: Intriguing role of termination and antitermination", Proc. Natl. Acad. Sci. 105, 18159-18164 (2008). http://dx.doi.org/10.1073/pnas.0806084105

[15] M. H. Friedman, "Principles and Models of Biological Transport", Springer, Berlin (2008).

[16] R. K. P. Zia, J. J. Dong, and B. Schmittmann, "Modeling Translation in Protein Synthesis with TASEP: a Tutorial and Recent Developments", J. Stat. Physics 144, 405-428 (2011). http://dx.doi.org/10.1007/s10955-011-0183-1

[17] L. Saiz, "The physics of protein-DNA interaction networks in the control of gene expression", J. Phys.: Condens. Matter 24, 193102 (1-15) (2012).

[18] J. Howard, "Mechanics of Motor Proteins and the Cytoskeleton", Sinauer Associates, Sunderland, MA (2001).

[19] S. Katz, J. L. Lebowitz, H. Spohn, "Nonequilibrium steady states of stochastic lattice gas models of fast ionic conductors", J. Stat. Phys. 34, 497-537 (1984). 
http://dx.doi.org/10.1007/BF01018556

[20] J. Krug, H. Spohn, "Universality classes for deterministic surface growth", Phys. Rev. A 38, 42714283 (1988).

http://dx.doi.org/10.1103/PhysRevA.38.4271

[21] J. Krug, "Boundary-induced phase transitions in driven diffusive systems", Phys. Rev. Lett. 67, 1882-1885 (1991). http://dx.doi.org/10.1103/PhysRevLett.67.1882

[22] G. M. Schultz, "Exactly Solvable Models for Many-Body Systems Far from Equilibrium" In: C. Domb, J. L. Lebowitz, (eds.): Phase Transitions and Critical Phenomena, Vol. 19. Academic Press, London Sydney Tokyo (2001).

[23] B. Schmittmann and R. K. P. Zia, "Statistical Mechanics of Driven Diffusive Systems" In: C. Domb, J. L. Lebowitz, (eds.): Phase Transitions and Critical Phenomena, Vol. 17. Academic Press, London Sydney Tokyo (1995).

[24] L. P. Pitaevskii, "Rigorous results of nonequilibrium statistical physics and their experimental verification", Physics - Uspekhi 54, 625-632 (2011). http://dx.doi.org/10.3367/UFNe.0181.201106d.0647

[25] J. Brankov, N. Pesheva, N. Bunzarova, "TASEP on chains with a double chain section in the middle: Computer simulations and a simple theory", Phys. Rev. E 69, 066128 (1-13) (2004).

[26] E. Pronina, A. B. Kolomeisky, "Theoretical Investigation of Totally Asymmetric Exclusion Processes on Lattices with Junctions", J. Stat. Mech.: Theory Exp., P07010 (1-16) (2005).

[27] B. Embley, A. Parmeggiani, N. Kern, "HEXTASEP: dynamics of pinned domains for TASEP transport on a periodic lattice of hexagonal topology", J. Phys.: Condens. Matter 20, 295213 (1-8) (2008).

[28] B. Embley, A. Parmeggiani, N. Kern, "Understand- ing totally asymmetric simple-exclusion-process transport on networks: Generic analysis via effective rates and explicit vertices", Phys. Rev. E 80, 041128 (1-22) (2009).

[29] R. Wang, M. Liu, R. Jiang, "Theoretical investigation of synchronous totally asymmetric exclusion processes on lattices with multiple-input-singleoutput junctions", Phys. Rev. E 77, 051108 (1-8) (2008).

[30] L. J. Cook, R. K. P. Zia, and B. Schmittmann, "Competition between multiple totally asymmetric simple exclusion processes for a finite pool of resources", Phys. Rev. E 80, 031142 (1-12) (2009).

[31] L. J. Cook and R. K. P. Zia, "Competition for finite resources", J. Stat. Mech.: Theory Exp., P05008 (116) (2012).

[32] P. Greulich, L. Ciandrini, R. J. Allen, and M. Carmen Romano, "Mixed population of competing totally asymmetric simple exclusion processes with a shared reservoir of particles", Phys. Rev. E 85, 011142 (1-11) (2012).

[33] C. A. Brackley, L. Ciandrini, and M. Carmen Romano, "Multiple phase transitions in a system of exclusion processes with limited reservoirs of particles and fuel carriers", J. Stat. Mech.: Theory Exp., P03002 (1-23) (2012).

[34] T. Ezaki and K. Nishinari, "Exact stationary distribution of an asymmetric simple exclusion process with Langmuir kinetics and memory reservoirs", J. Phys. A: Math. Theor. 45, 185002 (1-10) (2012).

[35] J. Brankov and N. Bunzarova, "Finite-size scaling and universality for the totally asymmetric simpleexclusion process", Phys. Rev. E 71, 036130 (1-10) (2005).

[36] J. Brankov and N. Bunzarova, "Finite-size scaling and universality at non-equilibrium phase transitions revisited", J. Theor. Applied Mech. (Sofia) 36, 57-76 (2006). 